\title{
OASs in Defense of Mycobacterial Infection: Angels or Demons?
}

\author{
Weipan Zhang, Xiaojian Cao, Gang Cao and Xi Chen* \\ State Key laboratory of Agricultural Microbiology, College of Veterinary Medicine, \\ Huazhong Agricultural University, Wuhan 430070, P. R. China \\ *Corresponding author: chenxi419@mail.hzau.edu.cn \\ DOI: https://doi.org/10.21775/cimb.040.221
}

\begin{abstract}
The interaction between pattern-recognition receptors (PRRs) and pathogenassociated molecular patterns (PAMPs) induces type I interferon (IFN) responses. IFNs stimulates hundreds of genes to exert its biological effects. OASs are the members of IFN-stimulate genes (ISGs). Among them, OAS1 activates RNase L to cleave RNA viruses genome, OAS2 activates downstream immune signaling pathways of IFNs, OAS3 induces RNase $L$ to cut the genome of RNA virus and activate IFN I response to enhance the immune effect, and OASL inhibits the survival of RNA viruses by activating RIG-I signaling pathway but promotes the reproduction of DNA viruses by inhibiting the cGAS signaling pathway. However, the role of OASs in mycobacterial infection remains incomprehensible. In this review, we summarized the latest literature regarding the roles of OASs in mycobacterial infection.
\end{abstract}

\section{Introduction}

Mycobacterium tuberculosis ( $M$. tuberculosis) is an obligate intracellular pathogen, which is capable of maintaining long-term persistence in host by modulating its innate and adaptive immune response (Toledo Pinto et al., 2018). The mechanisms by which host immune response restricts $M$. tuberculosis replication and transmission are still unknown. M. tuberculosis invades and induces large amounts of cytokines secretion by macrophage such as interferon (IFN), IL-1 $\beta$ and TNF $\alpha$ (Leisching et al., 2017b). Although type II IFN has been proved to restrict the proliferation and persistence of $M$. tuberculosis, secretion of type I IFN by host cells in turn benefits its intracellular survival and replication (Ahmed et al., 2016; Leisching et al., 2017b). IFN-stimulating genes (ISGs) are involved in the host's immune responses against bacterial infection such as $M$. tuberculosis (Leisching et al., 2017b). Thus, understanding the role of IFN 
signaling pathway is critical to elucidate the pathogenesis of M. tuberculosis. 2'-5'oligoadenylate synthetases (OASs) is IFN-inducible proteins, consist of OAS1, OAS2, OAS3 and 2'-5'-oligoadenylate synthetases-like (OASL) (Hornung et al., 2014). OASs are associated with the secretion of type I IFN and plays completely different immune regulatory role during viral and bacterial infection (Ghosh et al., 2019). Herein, we aim to demonstrate a landscape of OASs regulating mechanisms during infection, which may further clarify the role of OASs in mycobacterial infection.

\section{Roles of OASs in controlling virus infection}

OAS1, 2, 3 but not OASL have synthetase activity and function in host's defense against viral infection (Zhu et al., 2015). OAS1 recognizes viral double-stranded RNA (dsRNA) by a 3'-single-stranded pyrimidine (3'-ssPy) motif and stimulates the oligomerization of ATP into 2',5'-linked oligoadenylates (2,5A). 2,5A binds to ribonuclease $L(R N a s e ~ L)$ to cleave RNA resulting in attenuated proliferation of virus (Carey et al., 2019; Sasaki et al., 2002; Vachon et al., 2015). OAS2 inhibits the replication of RNA viruses by enhancing type I IFN activated downstream immune responses (Mengmeng et al., 2017; Sheng et al., 2016). When RNAs of ZIKA virus are recognized by RIG-I and melanoma differentiation-associated gene 5 (MDA5), inducement of type I IFN activates Jak/STAT signaling pathway leading to ISGs transcription to play antiviral and immune regulatory roles (Liao et al., 2020). Moreover, studies have shown that when NOD2 binds to OAS2, the activity of RNase $L$ is enhanced (Dugan et al., 2009). During RNA virus infection, OAS3 induces RNase $L$ to cleave intracellular RNAs, which activates RIG-I and MDA5 via downstream signal adaptor Mitochondrial Antiviral Signaling Protein (MAVS) to enhance type I IFN response and inhibits RNA virus replication (Diner et al., 2013; Li et al., 2016; Yoneyama et al., 2004).

OASs are also involved in regulating diverse cellular functions such as the inducement of apoptosis, enhancement of IFN- $\alpha$ and IFN- $\beta$ signaling responses, immune cell receptor modulation and autophagy (Leisching et al., 2018). In the absence of viral nucleic acids, the activation of OASs can restrict protein synthesis and induce cell apoptosis through RNase $L$ cleavage and degradation of host mRNA (Kristiansen et al., 2010; Li et al., 2016). However, the effect of OAS1, 2, and 3 on DNA virus invasion is still unclear.

Human OASL is one of the ISG and its mouse ortholog is 2'-5'-oligoadenylate synthetases-like 1 (OASL1) and 2'-5'-oligoadenylate synthetases-like 2 (OASL2), sharing $70 \%$ and $48 \%$ amino acid sequence identity with human OASL respectively (Zhu et al., 2015). It has an N-terminal structural domain that is highly conserved among OAS family, but its C-terminal structure is completely different from other members $(R, 1998)$. The $C$-terminal has a unique structure consists of two ubiquitin-like domains (UBL) and an OAS-like structure dsRNA binding domain that can bind to dsRNA (Diner et al., 2013). Studies on OASL2 are relatively rare, but there are more in-depth studies on OASL1. In the early stages of RNA virus infection (Figure 1), OASL1 capture viral RNAs by stress granules and promoted efficient RIG-I-like Receptor (RLR) signaling (Kang et al., 2018). In addition, OASL1 can rapidly transfer into antiviral stress granules (avSGs) and 
interacted with MDA5, a cytosolic viral sensor, within avSGs promoting type I IFN signaling (Kang et al., 2018). While in the late stages of infection, OASL1 interacts with IRF7 transcripts to inhibit its translation and results in decreased IFN expression (Kang et al., 2018). The interaction between OASL and transcriptional repressor methyl $\mathrm{CpG}$ binding protein 1 (MBD1) was found to enhance RIG-I signaling (Manzanillo et al., 2012; Watson et al., 2012). OASL binds directly to RIG-I in the cytosol and mimics polyubiquitin, leading to enhancement of antiviral signaling (Zhu et al., 2014). In addition, OASL can directly activate IFN- $\beta$ promoter, promoting the expression of IFN- $\beta$ to eliminate RNA virus (Oshiumi et al., 2015; Wang et al., 2018).

During DNA virus infection, OASL initials distinct immune response correspondingly from different RNA virus infection. When invading into host cells (Figure 1), DNA viruses could release DNA which may activate the enzyme activity of nucleotide transferase cGAS (Zevini et al., 2017). The activated cGAS facilitates ATP and GTP for the synthesis of 2'3'-cGAMP, which activates endoplasmic reticulum membrane protein STING (Chen et al., 2016). STING can be activated by subsequently cyclic dinucleotides (CDNs), then STING escorts tank binding kinase 1 (TBK1) to endosomal compartments to associate with and activate IRF3 and IRF7 that resulted in autophagy and type I IFN responses to limit the proliferation and spread of DNA viruses (Barber, 2014; Chen et al., 2016). While, OASL binding to cGAS results in the inhibition of cGAMP production without affecting its DNA-binding abilities, the interaction between OASL and cGAS is independent of the presence of DNA (Ghosh et al., 2019). Briefly, DNA viruses could induce an OASL-mediated type I interferon inhibitory effect by blocking cGAS-STING-TBK1 signaling pathway for its replication (Liu et al., 2017).

\section{Roles of OASs in controlling mycobacterial infection}

OASs are involved in defense against viral infection, but its function during $M$. tuberculosis infection remains elusive. Interestingly, the upregulation of OAS1, OAS2, and OAS3 expression was observed in both active and latent tuberculosis (Leisching et al., 2019). During active tuberculosis, OAS1 is the top up-regulated gene and its polymorphisms are associated with tuberculosis (Berry et al., 2010; Wu et al., 2018). When interferes the expression of OAS1, the number of viable intracellular $M$. tuberculosis significantly increased, and the expression of TNF- $\alpha$ and IL-1 $\beta$ decreased significantly relative to control (Berry et al., 2010; Kristiansen et al., 2010; Leisching et al., 2019). In addition, OAS1 expression was further detected in peripheral blood leukocytes from leprosy patients (Rego et al., 2018). OAS1 is induced and released to sera during $M$. leprae and $M$. tuberculosis infection (Rego et al., 2018). OAS2 expression is enhanced significantly during $M$. tuberculosis invasion (Mvubu et al., 2016). Similarly, when interferes the expression of OAS2 or OAS3, the number of viable intracellular M. tuberculosis significantly increased. The secretion of TNF- $\alpha$, Monocyte Chemoattractant Protein-1 (MCP-1), and IL-1 $\beta$ were reduced following OAS3 silencing in response to pathogenic mycobacteria infection, but only IL-1 $\beta$ levels were also decreased in the case of non-pathogenic mycobacterial infection (Leisching et al., 2019). Besides, the secretion of OAS1, 2, and IL-10 was not affected after OAS3 was 
silenced (Leisching et al., 2019). All three OAS i.e. 1, 2, and 3 are antagonists of intracellular $M$. tuberculosis replication by regulating cytokine secretion (Leisching et al., 2019).

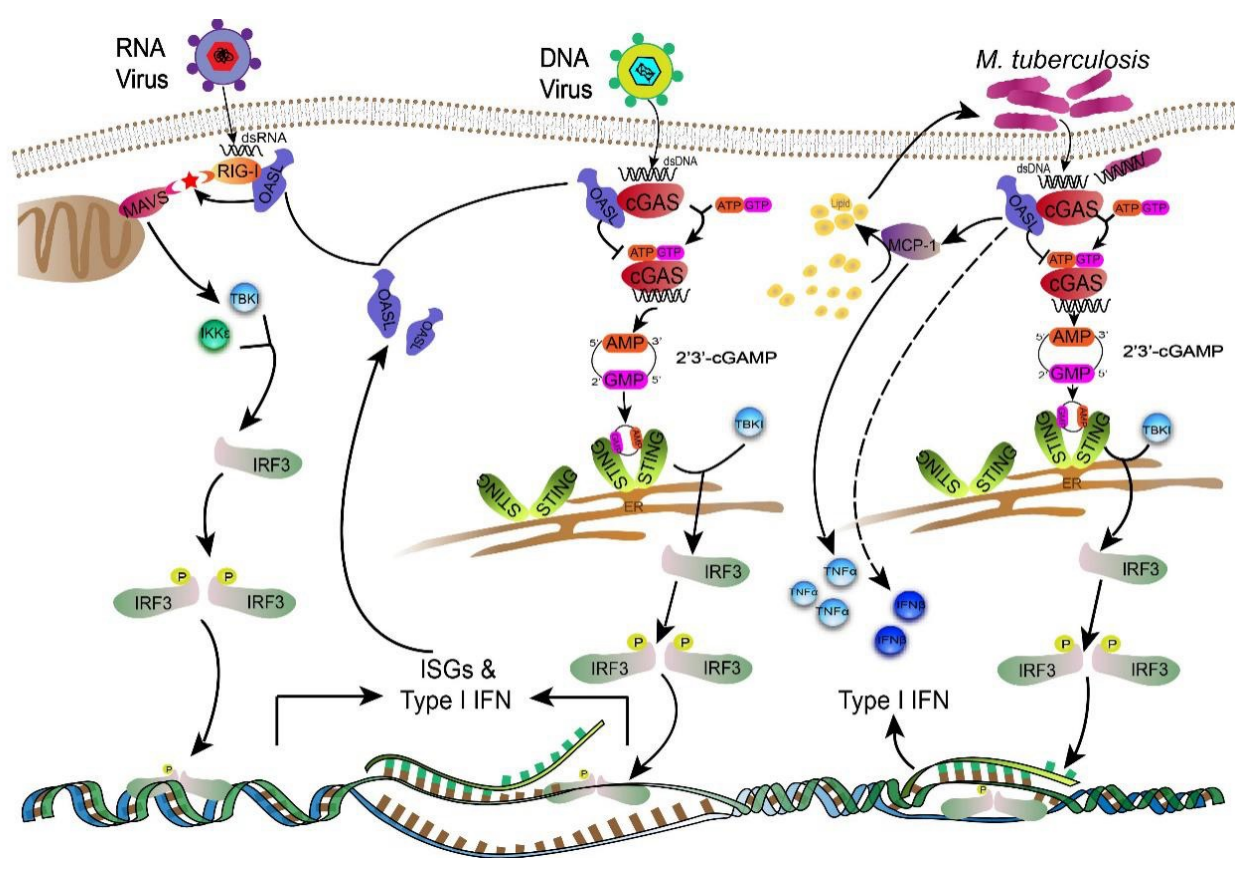

Figure 1. OASL initiates various anti-pathogen immune responses. When RNA virus enters the cell, the releasing of dsRNA activates RIG-I, OASL promotes RIG-I recognition of the virus, leads to the recognition of the virus, and activates the MAVS to promote the release of TBK1 and IKK enzymes. TBK1 initiates phosphorylation of IRF3, thus promotes the type 1 IFN response and finally lead to the clearance of the virus. When DNA virus enters the cell and releases dsDNA, which activates DNA sensor cGAS, catalyzes the synthesis of two phosphodiester bonds between GMP and AMP to produce 2'3'-cGAMP. Afterward, STING on the endoplasmic reticulum is activated by 2'3'-cGAMP, the activation of STING stimulates TBK1 and promoting type 1 IFN response and related ISGs. However, OASL inhibits cGAS's utilization of ATP and GTP, leading to virus proliferation and escape from being eliminated by the host immune response. During $M$. tuberculosis infection, the released dsDNA could be sensed by cGAS leading to autophagy induction and type I IFN production through CGAS-STING-TBK1 axis. Moreover, OASL directly activates MCP-1, and the activated MCP-1 will collect and aggregate lipid droplets in the cell, providing proper conditions for the survival of mycobacterium, thus promoting mycobacterium growth.

As the intracellular parasite, $M$. tuberculosis could escape into cytosol and the escaping $M$. tuberculosis is usually accompanied by the release of its DNA (Manzanillo et al., 2012). The extracellular mycobacterial DNA activates the DNAdependent cytosolic surveillance pathway (Figure 1) (Manzanillo et al., 2012; 
Watson et al., 2012). When M. tuberculosis escapes into cytosol, cGAS and mycobacterial DNA were co-located and aggregated (Wassermann et al., 2015). This interaction initiates cGAS utilization of GTP and ATP to produce cGAMP, which activates the STING-TBK1-IRF3 signaling axis and induces type I interferon production and autophagy activation (Barber, 2014; Majlessi and Brosch, 2015; Watson et al., 2015). cGAS is participating in intracellular $M$. tuberculosis role in balancing immune-protection and immune-pathogenesis (Majlessi and Brosch, 2015). It has been reported that OASL could bind to cGAS and inhibit IFN- $\beta$ production (Ghosh et al., 2019). During infection of $M$. tuberculosis, OASL is highly induced in host cells (Etna et al., 2015; Leisching et al., 2017a; Mvubu et al., 2016; Zhang et al., 2019). When OASL expression is suppressed, the number of both pathogenic and non-pathogenic mycobacteria was found increased compared to control, indicating that OASL is able to inhibit mycobacterial intracellular replication (Leisching et al., 2020). In addition, TNF- $\alpha$ and IL-1 $\beta$ secretion were significantly reduced along with decreasing MCP-1 production when interfering with OASL expression in macrophages (Leisching et al., 2020; Nancy et al., 2014). Decreased MCP-1 and TNF- $\alpha$ in turn lead to unrestricted growth and dissemination of mycobacteria (Hasan et al., 2006). MCP-1 provides an environment conducive to mycobacterium survival by ingesting and collecting lipid droplets (Figure 1) (Chetan et al., 2015; Koul et al., 2004). OASL can be detected in serum from active tuberculosis patients and with higher expression levels relative to latent tuberculosis patients (Sambarey et al., 2017). And the expression level significantly decreased after six months of IFN treatment, indicating that type I interferon induced OASL involved in controlling the growth of $M$. tuberculosis (Sambarey et al., 2017).

Similarly, M. leprae infection induced a high level of OASL in primary human Schwann cells, macrophages, and monocytes. The expression of OASL could be induced by mycobacterial genomic DNA in a STING dependent manner and inhibits autophagy for bacterial survival (de Toledo-Pinto et al., 2016). When OASL expression is inhibited, the viability of $M$. leprae was decreased. OASL can also affect the release of MCP-1 in the $M$. leprae that MCP-1 expression increases after silencing OASL expression (Rego et al., 2018). However, the expression of OASL decreased after BCG treatment and in response, the expression of MCP-1 increased (Rego et al., 2018). The study indicated that OASL actually promotes the survival of $M$. Leprae. M. leprae usually alters the host immune system by boosting lipid metabolism or suppressing proinflammatory cytokines, which are very similar to the host colonization mechanisms of $M$. tuberculosis (Guerreiro et al., 2013; Toledo Pinto et al., 2018). $M$. leprae suppress host antimicrobial responses are achieved through type I IFN induced IL-10 response, by causing binding of IL-27 with IFN- $\beta$, which leads to the inducement of IL-10 production (Teles et al., 2015). IL-10 exerts profound inhibition on innate and adaptive immunity, contributing to the chronic progressive diseases with bacterial infections and enabling $M$. leprae to maintain long-term persistence in the host (Teles et al., 2015). This finding suggests that OASL may no longer be associated solely with antiviral responses, but also involved in the antimycobacterial responses and host defense mechanisms in the macrophage. 
Altogether, it is possible that OASL manipulates mycobacterium replication in dissimilar ways.

\section{Conclusion}

Type I IFN signaling pathway serves as a positive role in regulation of $M$. tuberculosis intracellular survival. So far, it is known that OAS1, 2, 3 inhibit the survival and replication of $M$. tuberculosis by regulating the secretion of cytokines. It is noteworthy that the antagonistic effect of OASL in the treatment of $M$. tuberculosis but promotes the survival of $M$. leprae that may through inhibiting the cGAS pathway, and the more detailed mechanism remains to be studied.

\section{Acknowledgement}

This work is supported by the National Natural Science Foundation of China (grant no.31602061, 31872470).

\section{Competing interests}

The authors declare that they have no competing interests.

\section{References}

Ahmed, A., Nayak, S., Babu, S., and Vyakarnam, A. (2016). Impact of Type I IFN dysregulation in $M$. tuberculosis infection on $\mathrm{T}$ cell responses. International Journal of Infectious Diseases 45.

Barber, G.N. (2014). STING-dependent cytosolic DNA sensing pathways. Trends Immunol 35, 88-93.

Berry, M.P., Graham, C.M., McNab, F.W., Xu, Z., Bloch, S.A., Oni, T., Wilkinson, K.A., Banchereau, R., Skinner, J., Wilkinson, R.J., et al. (2010). An interferoninducible neutrophil-driven blood transcriptional signature in human tuberculosis. Nature 466, 973-977.

Carey, C.M., Govande, A.A., Cooper, J.M., Hartley, M.K., Kranzusch, P.J., and Elde, N.C. (2019). Recurrent Loss-of-Function mutations reveal costs to OAS1 antiviral activity in primates. Cell Host Microbe 25, 336-343 e334.

Chen, Q., Sun, L., and Chen, Z.J. (2016). Regulation and function of the cGASSTING pathway of cytosolic DNA sensing. Nat Immunol 17, 1142-1149.

Chetan, S., Lin, L., J, S.T., Glenna, P., David, F., Nicole, F., C, D.S., Branch, M.D., Jacques, P., Martine, G., et al. (2015). T Cell Responses against mycobacterial lipids and proteins are poorly correlated in south African adolescents. Journal of immunology (Baltimore, Md : 1950) 195.

de Toledo-Pinto, T.G., Ferreira, A.B., Ribeiro-Alves, M., Rodrigues, L.S., BatistaSilva, L.R., Silva, B.J., Lemes, R.M., Martinez, A.N., Sandoval, F.G., AlvaradoArnez, L.E., et al. (2016). STING-Dependent 2'-5' Oligoadenylate SynthetaseLike production is required for intracellular mycobacterium leprae Survival. J Infect Dis 214, 311-320.

Diner, E.J., Burdette, D.L., Wilson, S.C., Monroe, K.M., Kellenberger, C.A., Hyodo, M., Hayakawa, Y., Hammond, M.C., and Vance, R.E. (2013). The innate immune DNA sensor cGAS produces a noncanonical cyclic dinucleotide that activates human STING. Cell Rep 3, 1355-1361.

Dugan, J.W., Albor, A., David, L., Fowlkes, J., Blackledge, M.T., Martin, T.M., Planck, S.R., Rosenzweig, H.L., Rosenbaum, J.T., and Davey, M.P. (2009). 
Nucleotide oligomerization domain-2 interacts with 2'-5'-oligoadenylate synthetase type 2 and enhances RNase-L function in THP-1 cells. Molecular Immunology 47, 560-566.

Etna, M.P., Giacomini, E., Pardini, M., Severa, M., Bottai, D., Cruciani, M., Rizzo, F., Calogero, R., Brosch, R., and Coccia, E.M. (2015). Impact of mycobacterium tuberculosis RD1-locus on human primary dendritic cell immune functions. Sci Rep 5, 17078.

Ghosh, A., Shao, L., Sampath, P., Zhao, B., Patel, N.V., Zhu, J., Behl, B., Parise, R.A., Beumer, J.H., O'Sullivan, R.J., et al. (2019). Oligoadenylate-SynthetaseFamily protein OASL inhibits activity of the DNA sensor cGAS during DNA virus infection to limit interferon production. Immunity 50, 51-63 e55.

Guerreiro, L.T., Robottom-Ferreira, A.B., Ribeiro-Alves, M., Toledo-Pinto, T.G., Rosa Brito, T., Rosa, P.S., Sandoval, F.G., Jardim, M.R., Antunes, S.G., Shannon, E.J., et al. (2013). Gene expression profiling specifies chemokine, mitochondrial and lipid metabolism signatures in leprosy. PLoS One 8, e64748.

Hasan, Z., Jamil, B., Zaidi, I., Zafar, S., Khan, A.A., and Hussain, R. (2006). Elevated serum CCL2 concomitant with a reduced mycobacterium-induced response leads to disease dissemination in leprosy. Scand $\mathrm{J}$ Immunol 63, 241-247.

Hornung, V., Hartmann, R., Ablasser, A., and Hopfner, K.P. (2014). OAS proteins and cGAS: unifying concepts in sensing and responding to cytosolic nucleic acids. Nat Rev Immunol 14, 521-528.

Kang, J.S., Hwang, Y.S., Kim, L.K., Lee, S., Lee, W.B., Kim-Ha, J., and Kim, Y.J. (2018). OASL1 traps viral RNAs in stress granules to promote antiviral responses. Mol Cells 41, 214-223.

Koul, A., Herget, T., Klebl, B., and Ullrich, A. (2004). Interplay between mycobacteria and host signalling pathways. Nat Rev Microbiol 2, 189-202.

Kristiansen, H., Scherer, C.A., McVean, M., ladonato, S.P., Vends, S., Thavachelvam, K., Steffensen, T.B., Horan, K.A., Kuri, T., Weber, F., et al. (2010). Extracellular 2'-5' oligoadenylate synthetase stimulates RNase Lindependent antiviral activity: a novel mechanism of virus-induced innate immunity. J Virol 84, 11898-11904.

Leisching, G., Ali, A., Cole, V., and Baker, B. (2020). 2'-5'-Oligoadenylate synthetase-like protein inhibits intracellular $M$. tuberculosis replication and promotes proinflammatory cytokine secretion. Mol Immunol 118, 73-78.

Leisching, G., Cole, V., Ali, A.T., and Baker, B. (2019). OAS1, OAS2 and OAS3 restrict intracellular $M$. tb replication and enhance cytokine secretion. Int J Infect Dis 80S, S77-S84.

Leisching, G., Pietersen, R.D., van Heerden, C., van Helden, P., Wiid, I., and Baker, B. (2017a). RNAseq reveals hypervirulence-specific host responses to M. tuberculosis infection. Virulence 8, 848-858.

Leisching, G., Wiid, I., and Baker, B. (2017b). The association of OASL and Type I Interferons in the pathogenesis and survival of intracellular replicating bacterial species. Front Cell Infect Microbiol 7, 196.

Leisching, G., Wiid, I., and Baker, B. (2018). OAS1, 2, and 3: significance during active tuberculosis? J Infect Dis 217, 1517-1521.

Li, Y., Banerjee, S., Wang, Y., Goldstein, S.A., Dong, B., Gaughan, C., Silverman, R.H., and Weiss, S.R. (2016). Activation of RNase $L$ is dependent on OAS3 
expression during infection with diverse human viruses. Proc Natl Acad Sci U S A 113, 2241-2246.

Liao, X., Xie, H., Li, S., Ye, H., Li, S., Ren, K., Li, Y., Xu, M., Lin, W., Duan, X., et al. (2020). 2', 5'-Oligoadenylate Synthetase 2 (OAS2) inhibits Zika virus replication through activation of Type I IFN signaling pathway. Viruses 12, 418.

Liu, B., Tang, L., Zhang, X., Ma, J., Sehgal, M., Cheng, J., Zhang, X., Zhou, Y., Du, Y., Kulp, J., et al. (2017). A cell-based high throughput screening assay for the discovery of cGAS-STING pathway agonists. Antiviral Res 147, 37-46.

Majlessi, L., and Brosch, R. (2015). Mycobacterium tuberculosis meets the cytosol: the role of cGAS in anti-mycobacterial immunity. Cell Host Microbe 17, 733-735.

Manzanillo, P.S., Shiloh, M.U., Portnoy, D.A., and Cox, J.S. (2012). Mycobacterium tuberculosis activates the DNA-dependent cytosolic surveillance pathway within macrophages. Cell Host Microbe 11, 469-480.

Mengmeng, Z., Bo, W., Huawei, L., Jian, H., Xinxin, C., Linjian, W., Yinbiao, W., Sha, X., Songlin, Q., and Gaiping, Z. (2017). Porcine 2', 5'-oligoadenylate synthetase 2 inhibits porcine reproductive and respiratory syndrome virus replication in vitro. Microbial pathogenesis 111.

Mvubu, N.E., Pillay, B., Gamieldien, J., Bishai, W., and Pillay, M. (2016). Mycobacterium tuberculosis strains exhibit differential and strain-specific molecular signatures in pulmonary epithelial cells. Dev Comp Immunol 65, 321-329.

Nancy, H.J., Meenakshi, N., and D, D.S. (2014). Neutrophils from pulmonary tuberculosis patients show augmented levels of chemokines MIP-1a, IL-8 and MCP-1 which further increase upon in vitro infection with mycobacterial strains. Human immunology 75.

Oshiumi, H., Miyashita, M., Okamoto, M., Morioka, Y., Okabe, M., Matsumoto, M., and Seya, T. (2015). DDX60 is involved in RIG-I-Dependent and independent antiviral responses, and its function is attenuated by Virus-Induced EGFR activation. Cell Rep 11, 1193-1207.

R, H. (1998). p59OASL, a 2'-5' oligoadenylate synthetase like protein: a novel human gene related to the 2 '-5' oligoadenylate synthetase family. Nucleic acids research 18.

Rego, J.L., de Lima Santana, N., Machado, P.R.L., Ribeiro-Alves, M., de ToledoPinto, T.G., Castellucci, L.C., and Moraes, M.O. (2018). Whole blood profiling of leprosy type 1 (reversal) reactions highlights prominence of innate immune response genes. BMC Infect Dis 18, 422.

Sambarey, A., Devaprasad, A., Mohan, A., Ahmed, A., Nayak, S., Swaminathan, S., D'Souza, G., Jesuraj, A., Dhar, C., Babu, S., et al. (2017). Unbiased identification of blood-based biomarkers for pulmonary tuberculosis by modeling and mining molecular interaction networks. EBioMedicine 15, 112-126.

Sasaki, K., Fujita, I., Hamasaki, Y., and Miyazaki, S. (2002). Differentiating between bacterial and viral infection by measuring both C-reactive protein and 2'-5'-oligoadenylate synthetase as inflammatory markers. J Infect Chemother 8, 76-80.

Sheng, Z., Dan, Z., Xue, L., Weiting, L., Ruibing, C., and Puyan, C. (2016). Porcine 2', 5'-oligoadenylate synthetases inhibit Japanese encephalitis virus replication in vitro. Journal of medical virology 88 . 
Teles, R.M.B., Kelly-Scumpia, K.M., Sarno, E.N., Rea, T.H., Ochoa, M.T., Cheng, G., and Modlin, R.L. (2015). IL-27 Suppresses antimicrobial activity in human leprosy. J Invest Dermatol 135, 2410-2417.

Toledo Pinto, T.G., Batista-Silva, L.R., Medeiros, R.C.A., Lara, F.A., and Moraes, M.O. (2018). Type I Interferons, autophagy and host metabolism in leprosy. Front Immunol 9, 806.

Vachon, V.K., Calderon, B.M., and Conn, G.L. (2015). A novel RNA molecular signature for activation of 2'-5' oligoadenylate synthetase-1. Nucleic Acids Res 43, 544-552.

Wang, R., Yu, Y., Kong, W., Li, C., Kang, Y., Wang, G., Wang, W., He, J., and Zhao, M. (2018). Molecular cloning of porcine 2',5'-oligoadenylate synthetaselike protein and its role in porcine reproductive and respiratory syndrome virus infection. Microb Pathog 125, 281-289.

Wassermann, R., Gulen, M.F., Sala, C., Perin, S.G., Lou, Y., Rybniker, J., SchmidBurgk, J.L., Schmidt, T., Hornung, V., Cole, S.T., et al. (2015). Mycobacterium tuberculosis differentially activates cGAS- and Inflammasome-Dependent intracellular immune responses through ESX-1. Cell Host Microbe 17, 799-810.

Watson, R.O., Bell, S.L., MacDuff, D.A., Kimmey, J.M., Diner, E.J., Olivas, J., Vance, R.E., Stallings, C.L., Virgin, H.W., and Cox, J.S. (2015). The cytosolic sensor cGAS detects mycobacterium tuberculosis DNA to induce Type I Interferons and activate autophagy. Cell Host Microbe 17, 811-819.

Watson, R.O., Manzanillo, P.S., and Cox, J.S. (2012). Extracellular M. tuberculosis DNA targets bacteria for autophagy by activating the host DNAsensing pathway. Cell 150, 803-815.

Wu, S., Wang, Y., Chen, G., Zhang, M., Wang, M., and He, J.Q. (2018). 2'-5'Oligoadenylate synthetase 1 polymorphisms are associated with tuberculosis: a case-control study. BMC Pulm Med 18, 180.

Yoneyama, M., Kikuchi, M., Natsukawa, T., Shinobu, N., Imaizumi, T., Miyagishi, M., Taira, K., Akira, S., and Fujita, T. (2004). The RNA helicase RIG-I has an essential function in double-stranded RNA-induced innate antiviral responses. Nat Immunol 5, 730-737.

Zevini, A., Olagnier, D., and Hiscott, J. (2017). Crosstalk between cytoplasmic RIG-I and STING sensing pathways. Trends Immunol 38, 194-205.

Zhang, Y.W., Lin, Y., Yu, H.Y., Tian, R.N., and Li, F. (2019). Characteristic genes in THP1 derived macrophages infected with mycobacterium tuberculosis H37Rv strain identified by integrating bioinformatics methods. Int J Mol Med.

Zhu, J., Ghosh, A., and Sarkar, S.N. (2015). OASL-a new player in controlling antiviral innate immunity. Curr Opin Virol 12, 15-19.

Zhu, J., Zhang, Y., Ghosh, A., Cuevas, R.A., Forero, A., Dhar, J., Ibsen, M.S., Schmid-Burgk, J.L., Schmidt, T., Ganapathiraju, M.K., et al. (2014). Antiviral activity of human OASL protein is mediated by enhancing signaling of the RIG-I RNA sensor. Immunity 40, 936-948. 
